\title{
Assessment of a Standardized ROS Production Profile in Humans by Electron Paramagnetic Resonance
}

\author{
Simona Mrakic-Sposta, ${ }^{1}$ Maristella Gussoni, ${ }^{1}$ Michela Montorsi, ${ }^{2,3}$ \\ Simone Porcelli, ${ }^{2,3}$ and Alessandra Vezzoli ${ }^{3}$ \\ ${ }^{1}$ Dipartimento di Fisiopatologia Medico-Chirurgica e dei Trapianti, Università di Milano, Via Fratelli Cervi 93, 20090 Segrate, Italy \\ ${ }^{2}$ Università Telematica S. Raffaele Roma, Via F. Daverio 7, 20122 Milan, Italy \\ ${ }^{3}$ Istituto di Bioimmagini e di Fisiologia Molecolare, Consiglio Nazionale delle Ricerche, Via Fratelli Cervi 93, 20090 Segrate, Italy
}

Correspondence should be addressed to Alessandra Vezzoli, alessandra.vezzoli@ibfm.cnr.it

Received 14 March 2012; Revised 6 June 2012; Accepted 7 June 2012

Academic Editor: Steve R. McAnulty

Copyright (C) 2012 Simona Mrakic-Sposta et al. This is an open access article distributed under the Creative Commons Attribution License, which permits unrestricted use, distribution, and reproduction in any medium, provided the original work is properly cited.

\begin{abstract}
Despite the growing interest in the role of reactive oxygen species (ROS) in health and disease, reliable quantitative noninvasive methods for the assessment of oxidative stress in humans are still lacking. EPR technique, coupled to a specific spin probe $(\mathrm{CMH}$ : 1-hydroxy-3-methoxycarbonyl-2,2,5,5-tetramethylpyrrolidine) is here presented as the method of choice to gain a direct measurement of ROS in biological fluids and tissues. The study aimed at demonstrating that, differently from currently available "a posteriori" assays of ROS-induced damage by means of biomolecules (e.g., proteins and lipids) spin-trapping EPR provides direct evidence of the "instantaneous" presence of radical species in the sample and, as signal areas are proportional to the number of excited electron spins, lead to absolute concentration levels. Using a recently developed bench top continuous wave system (e-scan EPR scanner, Bruker) dealing with very low ROS concentration levels in small ( $50 \mu \mathrm{L})$ samples, we successfully monitored rapid ROS production changes in peripheral blood of athletes after controlled exercise and sedentary subjects after antioxidant supplementation. The correlation between EPR results and data obtained by various enzymatic assays (e.g., protein carbonyls and thiobarbituric acid reactive substances) was determined too. Synthetically, our method allows reliable, quick, noninvasive quantitative determination of ROS in human peripheral blood.
\end{abstract}

\section{Introduction}

Reactive oxygen species (ROS) are a group of compounds endowed with high reactivity and short half-life because of their tendency to give or receive electrons to attain stability.

On the other side, cells are exposed to a large variety of ROS by means of both exogenous and endogenous sources. Nevertheless, despite the extremely strong exposure of our whole organism to ROS coming from exogenous sources, endogenous ROS play the most important and extensive role, since, in the time course of our life, each body cell is continuously exposed to them. The major responsible of ROS production are mitochondria [1]; enzymes are another endogenous source of ROS. While most enzymes can produce ROS as byproducts of their activity (xanthine oxidase is a clear example) some of them seem specifically designed to produce ROS: nitric oxide synthase yields NO radicals; NADPH oxidase complex utilizes electrons to produce superoxide radicals from oxygen molecules [2].

The continuous ROS efflux from endogenous and exogenous sources results in a uninterrupted and cumulative oxidative damage to cell components so altering a lot of cell functions.

The most vulnerable biological targets for oxidative damage are proteins, membrane lipids, and DNA. Indeed the most usually adopted techniques for oxidative stress quantification are based on the determination of specific end products of the damage resulting from the interaction of ROS just with these biological macromolecules $[3,4]$. However, all these methods return an indirect ROS determination, while the only technique capable of providing a direct freeradicals detection is electron paramagnetic resonance (EPR). 
As a matter of facts, also this technique is uncapable of a direct radical detection because of the short half-life of a radical with respect to the EPR time scale [5]. In order to overcome this gap, generally a trick is therefore used: a stable chemical compound traps the radical, becoming a radical in turn, but with a half-life compatible with the EPR time scale, and so, is EPR detectable. The used compounds are classified into two main groups: spin traps, not biological and to be used at high concentration, and spin probes, working in the same way but at lower concentration and higher efficiency [6]. Moreover, just by its nature, EPR is a quantitative technique, because the signals are proportional to the number of the excited electron spins. Thus, using a reference compound, absolute concentration levels can be attained.

Despite the great interest in measuring ROS in biology and medicine, EPR technique has not till now been widely used because of several technical and methodological problems $[5,7]$.

A new commercial EPR spectrometer (e-scan, Bruker) is able to overcome the gap, for biological and medical applications, as it operates at the common microwave frequency and deals with very low concentration levels (nanomolar) in small sample volumes (50 microliters), also responding to both easy portability and handling features. By principle it can be applied to many samples and biological environments, like cultured cells, organs or in vivo to animals or human blood. Up to now, blood is the most commonly used biological sample to measure antioxidant markers and oxidation products induced by ROS production in human studies, whereas muscle biopsy is generally ethically impractical due to its invasive nature. To further reduce the invasiveness of the technique, hence increasing its clinical and diagnostic potential, herein, we investigated the application of the radical-probe approach to the measurement of ROS formation in human capillary blood. To this aim, we tested our method to monitor ROS in peripheral blood of healthy human volunteers after implementing two treatments known to affect redox status.

As is well known, one way of imposing oxidative stress is by means of physical exercise [8]: a unique relationship with oxygen free-radical formation is established, so providing an excellent model for examining the dynamic balance between oxidative challenge and antioxidant defence machinery in biological systems [9]. Indeed physical exercise is known to increase the generation of $\operatorname{ROS}[10,11]$ in response to increased oxygen utilization causing a disturbance in the prooxidant/antioxidant balance in favour of the former which results in oxidative stress. On the other side of the same coin, antioxidant compounds may alter redox status too, by reducing ROS production $[12,13]$.

Aim of this study was to evaluate the efficacy of ROS generation assessment by a new mini-invasive procedure adopting radical probe EPR technique and altering redox status by two interventions, namely, exercise and antioxidant administration. In addition a correlation of the obtained EPR data to oxidative damage production, this latter measured by enzymatic assays of the principal biomarkers, during both at rest and after controlled physical exercise was attempted.
In particular, ex vivo formation of ROS, reflecting metabolic activity of blood cells and production of ROS in vivo, was analyzed in capillary blood using bench-top electron spin resonance spectrometer e-scan. On the other side, it is worth noting that several biomarkers are available to quantify the oxidatively modified macromolecules in biological samples, although none of them can alone adequately describe oxidative damage; therefore, several assays have been proposed in order to reliably monitor oxidative stress/ damages in biological specimens. As extensively reviewed [4], the assessment of thiobarbituric acid-reactive substances (TBARS) and protein carbonyl (PC) content are among the most widely employed assays used to determine lipid peroxidation and oxidative damage to proteins, respectively. For all these reasons a possible correlation between EPR results and TBARS and PC collected data was attempted.

\section{Materials and Methods}

2.1. Exercise Treatment. Eighteen $(n=18)$ male elite athletes (aged $19.70 \pm 1.16$ years; height $1.78 \pm 0.04 \mathrm{~m}$; body mass $77.65 \pm 6.97 \mathrm{~kg}$ ) from the Varese hockey team were recruited to participate in the study. The subjects visited the laboratory two times. On the first day, anthropometric measures were collected and an incremental test on treadmill (1\% of grade) up to voluntary exhaustion to assess gas exchange threshold (GET) and peak $\mathrm{O}_{2}$ uptake $\left(\mathrm{VO}_{2}\right.$ peak) was also performed. After six minutes of warmup exercise (according to the subject's estimated level of physical fitness), the speed was increased $1 \mathrm{~km} \cdot \mathrm{h}^{-1}$ every minute. The mean $\mathrm{VO}_{2}$ peak measured was $51.57 \pm 1.36 \mathrm{~mL} \cdot \mathrm{kg}^{-1} \cdot \mathrm{min}^{-1}$. On the second visit, at least seven days after, the subjects performed a 10minutes constant-load submaximal exercise (CLE) at heavyintensity and speed corresponding to a $\mathrm{VO}_{2}$ equal to $\sim 50 \%$ of the difference between GET and $\mathrm{VO}_{2}$ peak.

2.2. Antioxidant Treatment. In the acute investigation, ten $(n=10)$ healthy sedentary women (aged $48.80 \pm 5.32$ years; height $1.64 \pm 0.03 \mathrm{~m}$; body mass $56.81 \pm 10.35 \mathrm{~kg}$ ) were treated with R-thioctic acid $(1.6 \mathrm{~g})$.

2.3. Blood Sampling. Each subject reported to the laboratory at 9:00 a.m. after an overnight fast for blood sampling. Subjects, all nonsmokers, refrained from alcohol and caffeine consumption for at least $24 \mathrm{~h}$, and were asked not to perform any form of exercise for $48 \mathrm{~h}$ before testing. A written informed consent was signed by all participants after being informed of all risks, discomforts, and benefits involved in the study. Procedures were in accordance with the Declaration of Helsinki, and institutional review board approval was received for this study.

2.4. EPR Protocol for ROS Detection. The experimental protocol adopted for ROS detection is shown in Figure 1. For each subject, recruited for exercise procedure, capillary blood was taken from the fingertip before and after (immediately, 10 , and 20 minutes) a constant-load exercise. 


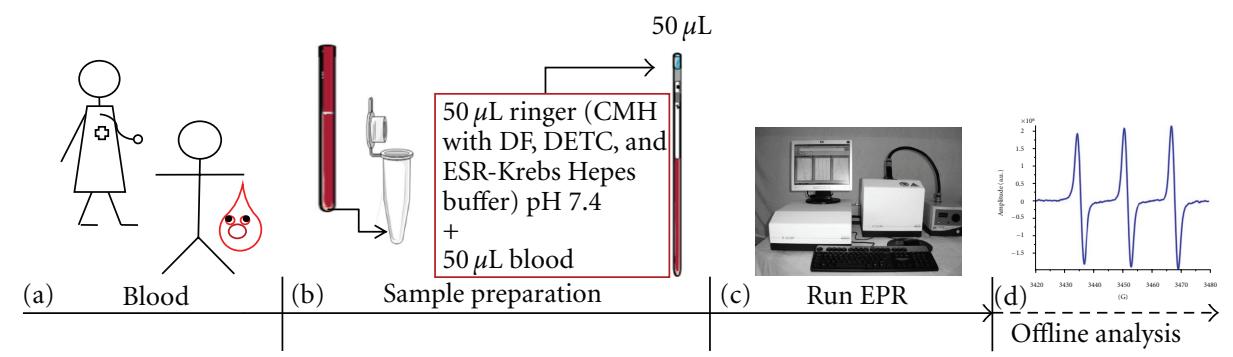

FIGURE 1: Step by step sketch of the EPR experimental protocol adopted to measure the ROS production by EPR.

In the antioxidant treatment, for each subject, capillary blood was drawn from the fingertip before and after R-thioctic acid administration (at 20, 40,60, 90 minutes, 2, and 3 hours). Control sampling at rest, at the same interval time, was carried out on the same subjects two days before supplementation.

For both experimental procedures, $50 \mu \mathrm{L}$ of blood, collected in heparinized capillary tubes (Cholestech LDX, Germany), were analyzed (Figure 1(a)). Among spin trapping (otherwise labelled probe) molecules, suitable for biological utilization, 1-hydroxy-3-methoxycarbonyl-2,2,5,5tetramethylpyrrolidine $(\mathrm{CMH}$, Noxygen Science Transfer \& Diagnostics, Germany) was adopted. A $1 \mathrm{mM} \mathrm{CMH}$ solution was prepared in buffer (Krebs-Hepes buffer (KHB) containing $25 \mu \mathrm{M}$ deferroxamine methane-sulfonate salt (DF) chelating agent and $5 \mu \mathrm{M}$ sodium diethyldithio-carbamate trihydrate (DETC)) at $\mathrm{pH}$ 7.4. Blood was immediately treated with $\mathrm{CMH}(1: 1) .50 \mu \mathrm{L}$ of the obtained solution was put in the glass EPR capillary tube (Noxygen Science Transfer \& Diagnostics, Germany), that was placed inside the cavity of the e-scan spectrometer (Bruker, Germany) for data acquisition (Figure 1(b)). The actual amount of solution analyzed was chosen to fill the entire sensitive area of the resonator cavity. Acquisition EPR parameters were: microwave frequency $=9.652 \mathrm{GHz}$; modulation frequency: $86 \mathrm{kHz}$; modulation amplitude: $2.28 \mathrm{G}$; center field: $3456.8 \mathrm{G}$; sweep width: $60 \mathrm{G}$; microwave power: $21.90 \mathrm{~mW}$; number of scans: 10 ; receiver gain: $3.17 \cdot 10^{1}$. Sample temperature was firstly stabilized and then kept at $37^{\circ} \mathrm{C}$ by the temperature and Gas controller "Bio III" unit, interfaced to the spectrometer. An example of the recorded EPR signal showing the triplet coming from the interaction of the ${ }^{14} \mathrm{~N}-\mathrm{OH}$ group of $\mathrm{CMH}$ with the ROS oxygen unpaired electron $\left(\mathrm{NOH}+\mathrm{O}_{2}^{*} \rightarrow\right.$ $\mathrm{NO}^{\bullet}+\mathrm{H}_{2} \mathrm{O}_{2}$ ) is displayed in Figure $1(\mathrm{~d})$. The radicals generated by the reaction of the probe with the blood radicals were acquired and the spectra sequentially recorded for about $5 \mathrm{~min}$ in order to calculate the ROS production rate. The EPR signal is proportional to the unpaired electron numbers and could, in turn, be transformed in absolute produced micromoles $\left(\mu \mathrm{mol} \cdot \mathrm{min}^{-1}\right)$ : the stable $\mathrm{CP}^{\bullet}$ (3Carboxy-2,2,5,5-tetramethyl-1-pyrrolidinyloxy) radical signal was recorded in a separate session and used as reference.

The high reproducibility of the EPR measurements is shown up in the plots reported in Figure 2. The data are referred to a couple of EPR measurement data (test I (open squares), test II (closed squares)) performed on blood capillary samples taken from the same healthy subject six hours apart. The data are expressed as arbitrary units and refer to EPR signal double integrals. The regression lines obtained from the collected data show an excellent correlation coefficient $\left(R^{2}=0.99\right)$ resulting in almost superimposable plots: test I (slope: 7.98, intercept: 15.49); test II (slope: 7.95, intercept: 15.95$)$. About $0.5 \%$ discrepancy between the ROS absolute production $\left(\mu \mathrm{mol} \cdot \mathrm{min}^{-1}\right)$ in the two tests was calculated.

2.5. Limits of Detection and Quantification with the Selected EPR Method. The limits of detection (LOD) and quantification (LOQ) can be estimated using the ICH Guidelines [14] that defines these parameters as the analytic concentrations at which the signal-noise ratios (SNR) are at least $3: 1$ and $10: 1$, respectively, and in EPR they depend upon the acquisition parameters, especially on the number of scans (NS), that influence linearly the SNR and the experimental time. In the EPR spectrum of a solution of ROS at known concentration $(6 \mu \mathrm{M})$ recorded under the same acquisition parameters adopted in the present study, the SNR of the line belonging to the ROS signal with NS = 10 was found to be 600. Therefore LOD and LOQ are immediately calculated as, respectively, $6 \mu \mathrm{M} \times 3 / 600=30 \cdot 10^{-3} \mu \mathrm{M}$ and $6 \mu \mathrm{M} \times 10 /$ $600=100 \cdot 10^{-3} \mu \mathrm{M}$.

2.6. Enzymatic Assays. Venus blood samples were taken at rest before and after (immediately, 20 minutes, 1, and 2 hour from the exercise end) constant-load submaximal exercise. Approximately $3 \mathrm{~mL}$ of blood were drawn from an antecubital vein, with subjects lying on a bed. The blood samples were collected in heparinized vacutainer tubes (Vacutainer, Becton Dickinson, USA), and plasma was separated by centrifuge (5702R, Eppendorf, Germany) at $1000 \mathrm{~g}$ for $10 \mathrm{~min}$ at $4^{\circ} \mathrm{C}$. The samples were then stored in multiple aliquots at $-80^{\circ} \mathrm{C}$ until assayed. Samples were thawed only once before analyses, which were performed within two weeks from collection.

2.6.1. Thiobarbituric Acid-Reactive Substances (TBARS). The measurement of TBARS is a well-established method to detect lipid peroxidation. We used TBARS assay kit (Cayman Chemical, USA) which allows a rapid photometric detection of the thiobarbituric acid malondialdehyde (TBAMDA) adduct at $532 \mathrm{~nm}$. Samples were read by a microplate reader 


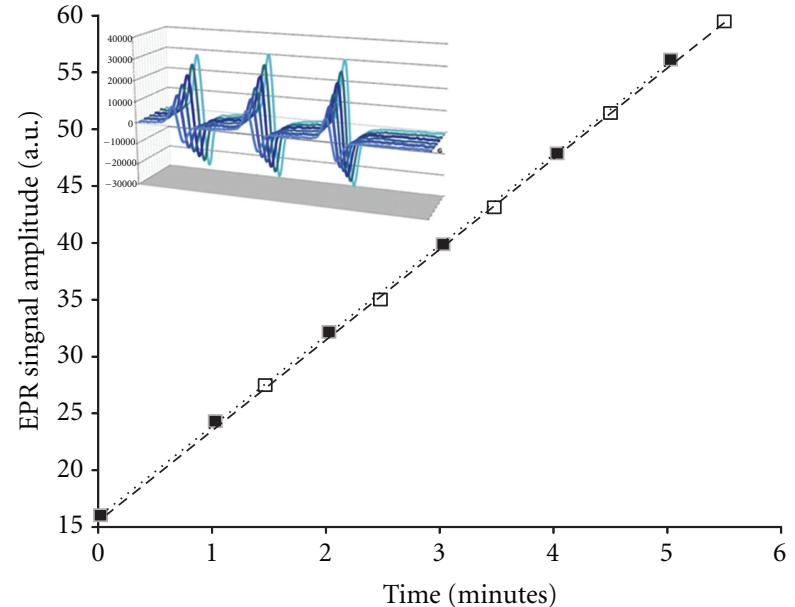

FIGURE 2: The high reproducibility of the EPR measurements is well demonstrated by the plots displayed in the figure showing the calculated EPR signal levels versus the elapsed time. Two tests were performed from a healthy subject taking 6 hours from each other. The best fitting straight lines $\left(R^{2}=0.99\right)$ were found almost superimposable: about the $0.5 \%$ discrepancy in the ROS absolute production rate $\left(\mu \mathrm{mol} \cdot \mathrm{min}^{-1}\right)$ was calculated between the measurements. The stacked plot of the recorded EPR spectra during a single experiment is displayed at the upper left corner. The spectra are centered at $g=1.997$. For each spectrum, the greatest signal amplitude difference in the triplet (arbitrary units) is returned by the acquisition routine, resulting in a point of the displayed graph. The ROS production rate (arbitrary units) is estimated by the best fitting line. It can be, in turn, converted in the absolute ROS production rate level $\left(\mu \mathrm{mol} \cdot \mathrm{min}^{-1}\right)$ throughout the acquisition of a stable radical compound like $\mathrm{CP}^{\bullet}$.

spectrophotometer (Infinite M200, Tecan, Austria). A linear calibration curve was computed from pure MDA-containing reactions. All samples were determined in duplicate and the interassay coefficient of variation was in the range indicated by the manufacturer (about 10\%).

2.6.2. Protein Carbonyls (PC). Reactive species produced directly or indirectly through lipid peroxidation intermediates also may oxidatively modify proteins. The accumulation of oxidized proteins was measured by content of reactive carbonyls. A Protein Carbonyl assay kit (Cayman Chemical, USA) was used to evaluate colorimetrically oxidized proteins. The samples were read at $370 \mathrm{~nm}$, by a microplate reader spectrophotometer (Infinite M200, Tecan, Austria), as described in detail by the manufacturer. Oxidized proteins values obtained were normalized to the total protein concentration in the final pellet (absorbance reading at $280 \mathrm{~nm}$ ), in order to consider protein loss during the washing steps, as suggested in the kit's user manual. All samples were determined in duplicate and the interassay coefficient of variation was in the range indicated by the manufacturer.

2.7. Data Analysis. All EPR spectra were obtained by using a software standardly supplied by Bruker (version 2.11, Win

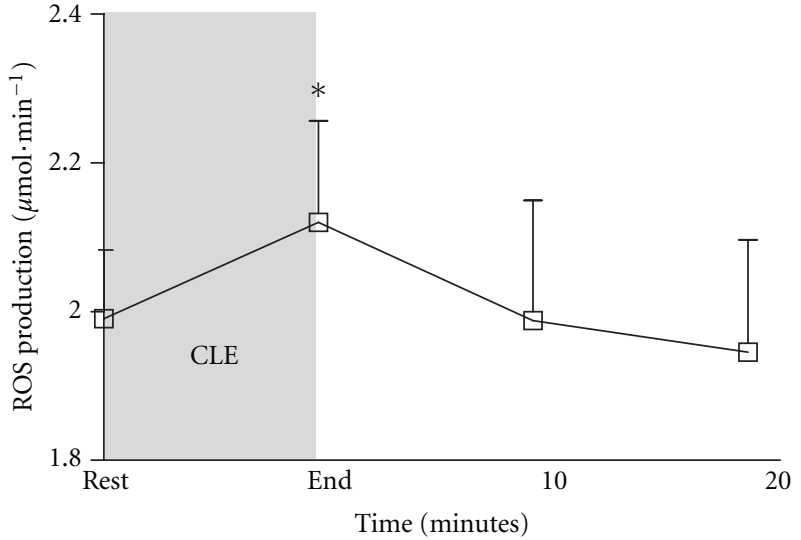

FIGURE 3: Time course of ROS production rate detected by EPR technique before (REST), immediately after the CLE (END) and at 10 and $20 \mathrm{~min}$ of recovery. Results are expressed as mean $\pm \mathrm{SD}$. Changes over time were significant at $P<0.05$ immediately post CLE compared to rest (*symbol).

EPR System). All spectra were collected by adopting the same protocol, as shown in Figure 1 and above reported.

Statistical analysis was performed using the GraphPad Prism package (GraphPad Prism 5, Software Inc. San Diego, CA). Data were analyzed using repeated Shapiro-Wilks $W$ tests. In the Shapiro-Wilk $W$ test, the null hypothesis is that the sample is taken from a normal distribution. This hypothesis is rejected if the critical value $P$ for the test statistic $W$ is less than 0.05. Experimental data were compared using one-way ANOVA with a Bonferroni post-hoc test. $P<0.05$ statistical significance level was accepted. All values were reported as means \pm standard deviation $(\mathrm{SD})$.

\section{Results}

3.1. Exercise. Exercise-induced EPR detectable enhancement in ex vivo ROS formation in capillary blood. The results are summarized in Figure 3. The kinetics of ROS production estimated by the EPR signal intensity levels variation at rest, immediately after CLE and during the 20 min of recovery after is shown.

Compared with resting data, a statistically significant $(P<0.05)$ increase of ROS production immediately at the end of CLE was observed, thereafter the ROS production returned to the preexercise condition.

At the same time, as can be observed in Figure 4(a), TBARS concentration increased immediately after exercise, significantly $(P<0.05)$ peaked 20 minutes after exercise and returned toward baseline levels thereafter.

Also PC concentration increased immediately after the end of CLE, even if showing a slower rate. Its level became statistically significant $(P<0.05)$ at 20 minutes after exercise, nevertheless the highest values were reached at 1 hour after the end of the exercise and declined thereafter (see Figure 4(b)). 


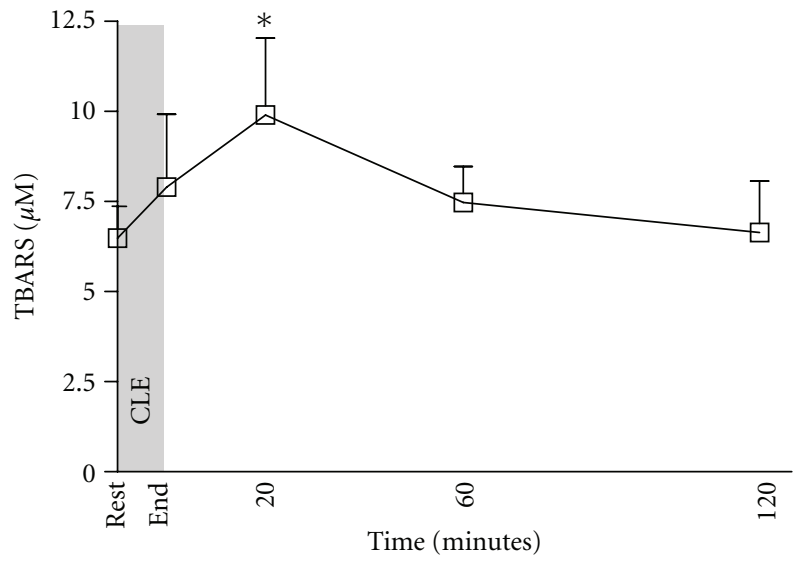

(a)

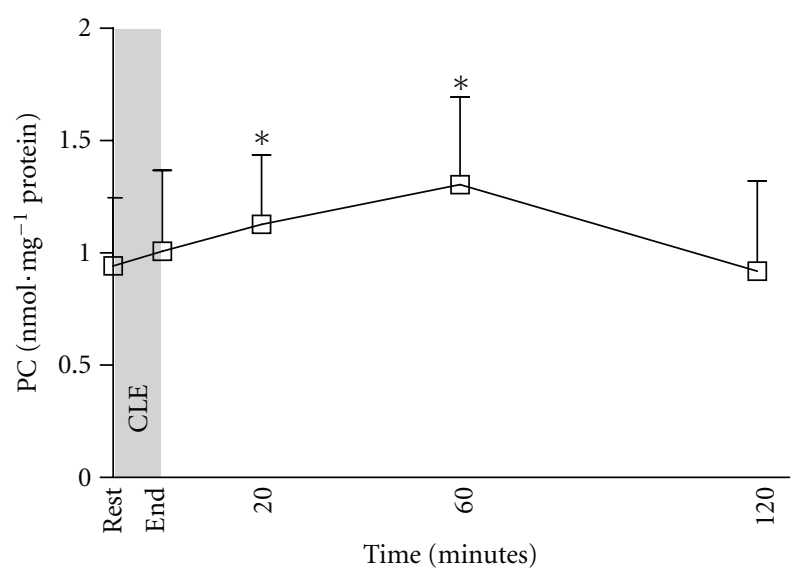

(b)

FIgURE 4: Time course of thiobarbituric acid reactive substances (a) and protein carbonyls (b) concentration before (REST), immediately after the CLE (END) and at $20 \mathrm{~min}, 1$, and 2 hours of recovery. Results are expressed as means \pm SD. Changes over time were significant at $P<0.05$ compared to rest ( ${ }^{*}$ symbol).

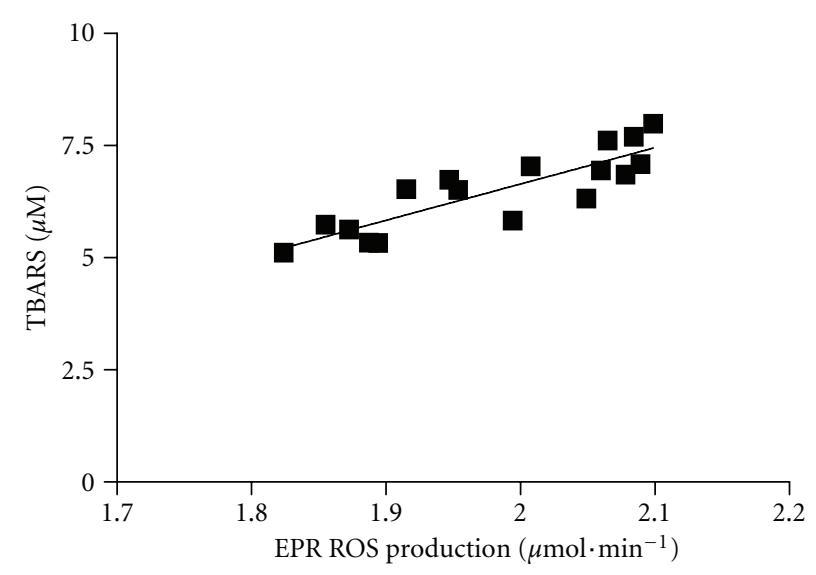

(a)

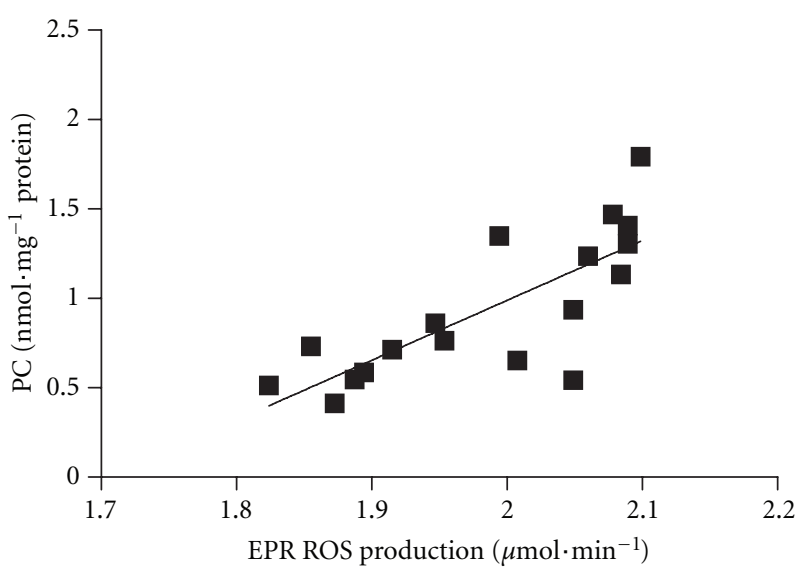

(b)

FIGURE 5: TBARS (a) and protein carbonyl PC (b) content as determined by enzymatic assays methods versus the ROS production rate $\left(\mu \mathrm{mol} \cdot \mathrm{min}^{-1}\right)$ calculated by EPR data (solid symbols). The linear regression lines (solid lines) are reported. The variance analysis (Pearson product-moment correlation) indicated a positive association for both TBARS and PC $\left(R^{2}\right.$ values $=0.74$ and $0.60, P<0.05$, resp.).

3.2. Correlation between ROS Production and Biomarkers of Oxidative Damage. Table 1 reports the mean values of plasma TBARS and PC and the ROS production values of capillary blood at rest. A positive relationship was found at rest between ROS production and plasma TBARS concentrations $\left(R^{2}=0.74, P<0.05\right)$ (Figure 5(a)) and with plasma PC concentrations $\left(R^{2}=0.60, P<0.05\right)$ (Figure 5(b)). At high-resting ROS production rate levels corresponded greater plasma TBARS and PC concentrations.

3.3. Antioxidant Supply. Antioxidant supplementation induced EPR detectable changes in formation of ex vivo ROS in capillary blood, the results are summarized in Figure 6. The kinetics of ROS production estimated by the EPR signal intensity at rest, immediately and during the 3 hours after Rthioctic acid administration is shown in the figure together
TABLE 1: Plasma TBARS and protein carbonyl levels and ROS production rate in capillary blood of hockey athletes at rest. Results are presented as mean $\pm \mathrm{SD}$.

\begin{tabular}{ll}
\hline TBARS $(\mu \mathrm{M})$ & $6.49 \pm 1.01$ \\
Protein carbonyls $\left(\mathrm{nmol} \cdot \mathrm{mg}^{-1}\right.$ protein $)$ & $0.94 \pm 0.40$ \\
ROS $\left(\mu \mathrm{mol} \cdot \mathrm{min}^{-1}\right)$ & $1.99 \pm 0.09$ \\
\hline
\end{tabular}

with the data recorded without supplementation at the corresponding interval time. The ROS production rate level, subsequent to the supply, increased after 20 minutes, then decreased (40 minutes) and kept at a lower level with respect to the resting value, reaching its lowest significant $(P<0.01)$ level 90 minutes after supplementation, then returning to the baseline. No significant difference was observed between 


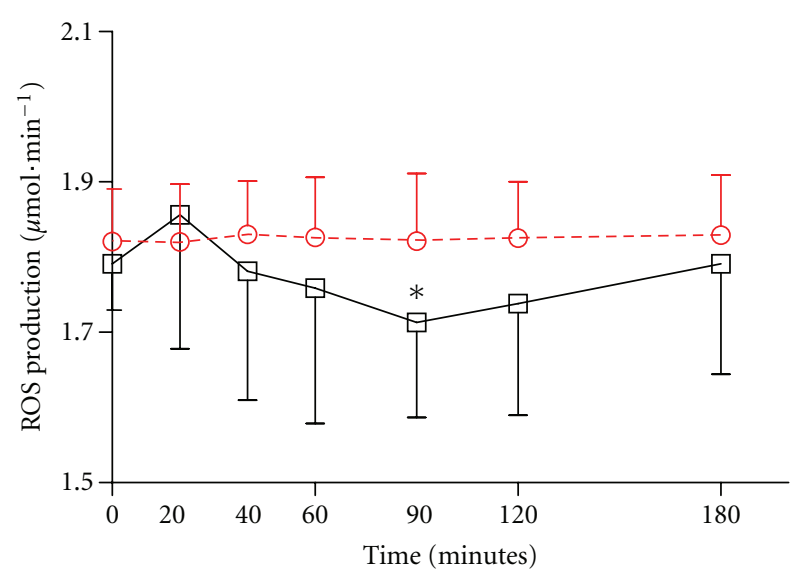

FIgURE 6: Time course of ROS production rate $\left(\mu \mathrm{mol} \cdot \mathrm{min}^{-1}\right) \mathrm{cal}-$ culated by the EPR acquisition data without supplementation (red circle) and following antioxidant (R-thioctic acid) supply (black square). Results are expressed as mean $\pm \mathrm{SD}$. Changes over time resulted significant $(P<0.01)$ at 90 min post, compared to pre-supplementation ( ${ }^{*}$ symbol).

experimental baseline and control data recorded without supplementation.

\section{Discussion}

Electron spin resonance/electron paramagnetic resonance (ESR/EPR) spectroscopy is, without any doubt, the only direct way to detect and measure free radicals. EPR spectroscopy can be used to directly study free radicals in tissues or tissue fractions [15], while spin-trapping agents can be used to stabilize free radicals and make them easier to detect.

However, the technique suffers of serious limitations, most notably a lack of sensitivity at the concentrations of free radicals typically found in biological systems.

The relatively simple blood withdrawal procedure, compared to the more invasive tissue biopsy, seems to be the dominant factor in the overwhelming use of blood measurements in human studies.

In this case, it is also important to recognize the limitations of spin trapping, which ultimately relies on the ex vivo detection of relatively stable species that are formed clearly downstream of the primary reaction pathway that we assume reflecting dynamic events in vivo.

Nevertheless, blood interacts with all organs and tissues and, consequently, with many possible sources of reactive species. In addition, a multitude of oxidizable substrates are already present in blood and carrying a multitude of substances that are considered oxidative stress markers (e.g., TBARS, protein carbonyls). Changes in the blood concentrations of these markers reflect corresponding changes in the tissue of interest (most of the time skeletal muscle) [16].

As a matter of facts, the vast majority of the relevant human studies have measured the redox status by using plasma or serum. This choice was probably adopted after considering that plasma better reflects tissue redox status together with the ease of plasma collecting procedure.
However, with this latter choice we cannot exclude potential artefacts generated by in vitro chemistry during the preparation and incubation phase of the spin probe or spin trap used, although this limitation is not exclusive to EPR sample preparation since it has interpretive implications for any reactive metabolite measured ex vivo, especially in human plasma.

Moreover, since the classic tests have mainly quantified the ROS levels in human plasma but not those associated with erythrocytes, these studies have not taken into account the role of circulating cells. Ginsburg et al. [17] raised serious doubts whether reports on antioxidant quantifications carried out exclusively in plasma can be trusted to represent true oxidant-scavenging abilities.

Indeed red blood cells may exert both antioxidant and prooxidant activity. Because of the high-iron concentration ( $\sim 20 \mathrm{mM})$, the red blood cell (RBC) can be considered an "iron mine" but, paradoxically, it is also one of the major components of blood antioxidant capacity and one of the most resistant to oxidative stress cell. A very efficient intracellular reducing machinery, coupled with its high-cell density makes the erythrocyte an effective "sink" of reactive species. Probably not only the blood per se but, more important, the whole organism can benefit from RBC scavenging ability. The reverse side of RBC antioxidant power is its capability of being, in turn, a source of reactive species. The superoxide radical generated within the RBC by deoxygenated or partially oxygenated hemoglobin, usually found at low levels and likely under physiologic conditions, does not represent a big hazard for the cell. Similarly, the ability of RBC to scavenge or generate nanomolar concentrations of $\mathrm{NO}^{\bullet}$ can be easily handled by the methemoglobin reductase/NADH/glycolysis system. Completely different may be the situation when the erythrocyte crosses a tissue where an intense production of reactive oxygen/nitrogen species is occurring. Under these conditions, the RBC may accumulate oxidative damage, in turn reflecting the oxidative stress of other tissues and organs. For this reason, oxidative status of RBCs is potential candidate for monitoring the overall oxidative stress status.

During exercise, since reactive species are generated by both blood and muscle, it is reasonable to assume that there is a bidirectional movement of reactive species from the muscle to the blood, and vice versa, until equilibrium is reached. The same may hold true for exchanges among blood constituents, namely, plasma, erythrocytes, leukocytes, and platelets [18], once that certain basic assumptions are met: reactive species with adequate half-life have the ability to cross membranes and generate reactive species at the vicinity of the compartments considered.

Based on all these considerations and aiming at reducing the invasiveness of the method and hence increasing its clinical and diagnostic potential, herein, we investigated the application of the radical-probe approach to the measurement of oxidative stress status in peripheral blood. Moreover fresh, rather than frozen, samples were used for the EPR measurements to be able to gain an estimation of the ROS production rate, instead of a single level, well assuming that this procedure allowed us to attain more precise and reliable results. 
Unpaired electron(s) carrying species are, as such, EPR visible. However ROS half-life (superoxide $\left[\mathrm{O}_{2}{ }^{-{ }^{-}}\right] t_{1 / 2}(\mathrm{~s})$ : $10^{-4}$; nitric oxide $\left[\mathrm{NO}^{\bullet}\right]: 0,4$ at ambient temperature) is too short if compared to the EPR time scale so they are EPR-invisible. Therefore the species have to be "trapped" and transformed to a more stable radical species to become EPR detectable. Among spin trapping or probe molecules, suitable for biological utilization, $\mathrm{CMH}$ was adopted, since it is a molecule capable of diffusion in all cell compartments, including mitochondria [6]. Indeed, due to its peculiar physical-chemical properties, $\mathrm{CMH}$ probe is able to cross biological membranes, thereby detecting ROS both in plasma and intracellular compartments. In this way, EPR measurements allowed us to attain a relative quantitative determination of ROS production rate in human blood samples.

In addition, owing to its high efficiency in radical detection, $\mathrm{CMH}$ probe can be used at very low concentrations $(0.5-1 \mathrm{mM})$ compared to spin traps $(10-50 \mathrm{mM})$, which minimizes side-effects of the probes on the biological samples. Moreover $\mathrm{CMH}$ rapidly reacts and allows radical detection in a single chemical reaction, while other probes require at least two reactions, which may cause artefacts [19].

The high reproducibility of the measurement was demonstrated by performing the experiments two times on the same subject six hours apart. In fact the procedure itself makes impossible to repeat the same experiment several times on the same blood sample. Nevertheless, the collected data suggested that repeated experiments from a resting subject gave almost superimposable results (see Figure 2 and red circle Figure 6).

Considerable evidence has linked exhaustive exercise with extensive free radicals formation. Davies et al. [8] were the first to establish exercise-induced free radicals formation after exhaustive physical activity by demonstrating a heightened EPR signal (around $g=2.004$ ) in muscle and liver homogenates. Other studies [20,21] have also been able to demonstrate heightened EPR signals with exercise. There are numerous reports that provide reasonable support to the notion that exercise increases the production of reactive oxygen species, and that mitochondria are important sources of these oxidants $[9,22]$. Other sources of oxidative stress during physical exercise are inflammatory responses mediated by neutrophils [23], the release of transition metals, such as iron, that supports Fenton chemistry with formation of hydroxyl radical, the interaction of metmyoglobin and methemoglobin with lipid peroxides [24], and the activity of xanthine oxidase [25], possibly within an ischemia-reperfusion model [26]. It may be surmised that high-intensity physical exercise disrupts the fragile balance between oxidants and antioxidant defences and that the mitochondrial respiratory chain and other pathways contribute to free radicals generation. As indicated above, blood is a tissue of paramount importance in regulating redox status changes appearing during exercise.

Using ESR spectroscopy, our study clearly demonstrated in human capillary blood that a short-term constant-load submaximal exercise (CLE) at heavy intensity, induced oxidative injuries since a significant increase in ROS production was detected (Figure 3).
As reported elsewhere [27], professional athletes showed a rapid increase in ROS on starting exercise. This was followed by a gradual decrease in the magnitude of the ROS production, reaching the initial value after $20 \mathrm{~min}$. This is in agreement with the concept that adaptive responses to aerobic training programs render athletes' enzymes less responsive over time to further significant activation, and that increased ROS generation caused by physical exercise overwhelms the capacity of the body to detoxify ROS.

Parenthetically, we would like to underline that main aim of the present study was to evaluate the efficacy of ROS generation assessment by adopting a mini invasive procedure. Just for this reason we have been forced to use arterial capillary blood samples even if we are completely aware that arterial blood, because of circulatory system design, is very far from muscles that are the main ROS sources during exercise. Therefore, we expect that when using a systemic determination the ROS detected levels will be surely lower mainly because part of the produced ROS have been buffered by blood itself. Bailey et al. [28] showed, in typical EPR spectra, a positive venoarterial concentration of $\alpha$-phenyltert-butylnitrone (PBN) adducts, detected in the arterial and venous circulation, at moderate intensity exercise. In the same paper the authors reported an increase in the EPR signal amplitude, according to the exercise intensity both in the arterial and venous circulation. Nevertheless the ROS detected level was always found lower in arterial with respect to venous blood samples. Indeed it is worth noting that, despite the moderate exercise levels adopted in the present study, the method was found suitable to detect a significant difference; demonstrating its reliability even under the adopted experimental conditions. On the other side, under the adopted acquisition protocol, the ROS levels measured in the present study resulted over the estimated limit of detection and quantification.

Increased production of reactive oxygen and nitrogen species after cessation of exercise may have contributed to elevated levels of lipid and protein peroxidation markers after exercise (Figure 4). The measurement of these latter only in plasma is enough to describe the changes in erythrocyte and muscle redox status because of a strong communication between the different compartments [16]. Indeed results from the present study indicate that lipid peroxidation, as measured by TBARS, was elevated immediately and until 20 min after exercise (Figure 4(a)). Many studies have provided indications for substantial increases in plasma lipid-peroxidation levels after aerobic exercise [29-31]. The delay in TBARS clearance, compared to more rapid ROS production kinetics, is in agreement with the observation of Echtay et al. [32] who proposed that lipid peroxidation products regulate mitochondrial ROS production by inducing the expression of proteins that inhibit mitochondrial $\mathrm{O}_{2}{ }^{\bullet}$ production by inducing uncoupling. Otherwise, removal of oxidized proteins from blood is, presumably, a time-consuming process, also considering that oxidative modifications in protein can occur by the effect of ROS directly or indirectly through conjugation to lipoxidation end products. For these reasons, protein carbonyls concentration remained elevated for a prolonged period (1 h) after exercise (Figure 4(b)). 
Other studies generally have reported increases similar to ours immediately after exercise, whereas the increases in protein carbonyls mostly disappeared after 0.5 to $6 \mathrm{~h}$ of recovery $[29,33-35]$. The different life response of protein carbonyls reported may be partly attributed also to the different intensity and more or less muscle-damaging exercise mode used in the studies and to differences in the physical fitness of the participants employed [36].

Secondary aim of this study was to examine whether measuring blood oxidative stress markers, a currently common practice in biomedical research, is indicative of ROSproduction. Although almost all oxidative stress biomarkers have been criticized for their reliability (including those used in the present study) [37, 38], it is apparent that, at rest, all changes indicating increased oxidative stress are directly related to ROS production (Figure 5). However the time-course changes of the used markers of oxidative stress were delayed and longer (Figures 4(a) and 4(b)) than ROSproduction kinetics (Figure 3 ), and no correlation is possible in dynamic conditions. In other words, it must be stressed that the good correlation found between EPR and enzymatic assays data do not represent a validation test for the here proposed EPR technique. By principle, the adopted enzymatic methods can quantitate the damage arising from ROS production, while EPR is the only technique capable, by using suitable probes, of quantitating the ROS production itself. These are the reasons why we could not hypothesize an "a priori" existing relationship between these methods we cannot absolutely affirm that ROS production will at the same time produce damage. On the other hand the data collected in the present study seem to suggest that the practice of the vast majority of the relevant studies to collect one blood sample immediately after or at some other early point postexercise can potentially lead to inaccurate deductions [29].

Similar consideration may be expressed for the analysis of the effects of antioxidant supplementation too. Thioctic acid ( $\alpha$-lipoic acid) was adopted for the study. In fact this molecule is an endogenous antioxidant which, in its reduced form, that is, dihydrolipoic acid, forms a thiol-disulphide redox system [39-41]. Moreover this moiety may also replenish intracellular GSH levels $[42,43]$ and is an essential cofactor for the multienzyme complexes and pyruvate dehydrogenase.

Indeed, in our study, the time corresponding to the peak activity of R-thioctic acid is delayed well after supplementation (Figure 6), as reported by other authors too [44]. Antioxidants may be defined as molecules that prevent cell damage against free radicals and are critical for maintaining optimum health in both animals and humans. In all living systems, cells require adequate levels of antioxidant defences in order to avoid the harmful effect of an excessive production of ROS, and so preventing the associated damage. Indeed excess of ROS production might play a role in pathophysiology of many disease conditions, including cancer, Alzheimer's disease, and atherosclerosis. Many basic research studies and observational epidemiologic studies in humans suggest that antioxidants can prevent oxidative damage. However, this is still a controversial issue because the results of clinical trials have been inconsistent [45]. Indeed there is a need for large multicentre prospective randomized control trials to assess the effects of different types and doses of antioxidant supplementation in selected groups of subjects. The methodological approach adopted in this study might be an efficient and practical tool to solve the question both in acute or chronic conditions.

\section{Conclusion}

Although the healthcare field is increasingly aware of the importance of free radicals and oxidative stress, screening and monitoring has not yet become a routine test. The method herein presented allows reliable, rapid, and noninvasive measurement of the instantaneous concentration of ROS directly in human peripheral blood. Due to its simplicity coupled with the high sensitivity and specificity of EPR spectroscopy, it compares favourably with the few currently available methods that have been successfully applied to measure radical species in human blood. After accurately testing and defining the most appropriate assay procedure, this kind of EPR determination can become an even turnkey, automated technique for a lot of routine and medical diagnostic applications on the time course of oxidative stress too. Moreover these findings seem to be valid both at rest and after interventions able to alter the redox status of a living system. In conclusion, ROS production assessed by the here proposed EPR measurement procedure in capillary blood seems to be well suitable to provide a reliable indication about the free radical-mediated changes that appear in skeletal muscle, heart, and liver.

\section{Acknowledgments}

The authors wish to thank the Scientific Commission of Italian Federation of Sport Medicine for the financial support. They are grateful to all the athletes and trainers of hockey Varese team and to all subjects that participated in the experimentation. The authors also thank Dr. Manuela Liberi and Dr. Roberto Melzi of Italian Bruker Biospin for their kind technical support.

\section{References}

[1] L. Gille and H. Nohl, "The ubiquinol/bcl redox couple regulates mitochondrial oxygen radical formation," Archives of Biochemistry and Biophysics, vol. 388, no. 1, pp. 34-38, 2001.

[2] S. K. Powers and M. J. Jackson, "Exercise-induced oxidative stress: cellular mechanisms and impact on muscle force production," Physiological Reviews, vol. 88, no. 4, pp. 1243-1276, 2008.

[3] A. E. Holley and K. H. Cheeseman, "Measuring free radical reactions in vivo," British Medical Bulletin, vol. 49, no. 3, pp. 494-505, 1993.

[4] R. Kohen and A. Nyska, "Oxidation of biological systems: oxidative stress phenomena, antioxidants, redox reactions, and methods for their quantification," Toxicologic Pathology, vol. 30, no. 6, pp. 620-650, 2002. 
[5] G. R. Buettner and B. A. Jurkiewicz, "Ascorbate free radical as a marker of oxidative stress: an EPR study," Free Radical Biology and Medicine, vol. 14, no. 1, pp. 49-55, 1993.

[6] S. I. Dikalov, I. A. Kirilyuk, M. Voinov, and I. A. Grigor'Ev, "EPR detection of cellular and mitochondrial superoxide using cyclic hydroxylamines," Free Radical Research, vol. 45, no. 4, pp. 417-430, 2011.

[7] N. Khan and H. Swartz, "Measurements in vivo of parameters pertinent to ROS/RNS using EPR spectroscopy," Molecular and Cellular Biochemistry, vol. 234-235, pp. 341-357, 2002.

[8] K. J. A. Davies, A. T. Quintanilha, G. A. Brooks, and L. Packer, "Free radicals and tissue damage produced by exercise," Biochemical and Biophysical Research Communications, vol. 107, no. 4, pp. 1198-1205, 1982.

[9] L. L. Ji, "Antioxidants and oxidative stress in exercise," Proceedings of the Society for Experimental Biology and Medicine, vol. 222, no. 3, pp. 283-292, 1999.

[10] D. M. Bailey, I. S. Young, J. McEneny et al., "Regulation of free radical outflow from an isolated muscle bed in exercising humans," American Journal of Physiology, vol. 287, no. 4, pp. H1689-H1699, 2004.

[11] D. A. Bailey, L. Lawrenson, J. McEneny et al., "Electron paramagnetic spectroscopic evidence of exercise-induced free radical accumulation in human skeletal muscle," Free Radical Research, vol. 41, no. 2, pp. 182-190, 2007.

[12] M. C. Gomez-Cabrera, E. Domenech, and J. Viña, "Moderate exercise is an antioxidant: upregulation of antioxidant genes by training," Free Radical Biology and Medicine, vol. 44, no. 2, pp. 126-131, 2008.

[13] B. Poljsak, "Strategies for reducing or preventing the generation of oxidative stress," Oxidative Medicine and Cellular Longevity, vol. 2011, Article ID 194586, 15 pages, 2011.

[14] ICH Harmonised Tripartite Guideline, "Validation on Analytical Procedures: Text and [Methodology]," Q2(R1), Step 4, 2005.

[15] S. Sachdev and K. J. A. Davies, "Production, detection, and adaptive responses to free radicals in exercise," Free Radical Biology and Medicine, vol. 44, no. 2, pp. 215-223, 2008.

[16] A. S. Veskoukis, M. G. Nikolaidis, A. Kyparos, and D. Kouretas, "Blood reflects tissue oxidative stress depending on biomarker and tissue studied," Free Radical Biology and Medicine, vol. 47, no. 10, pp. 1371-1374, 2009.

[17] I. Ginsburg, R. Kohen, and E. Koren, "Quantifying oxidantscavenging ability of blood," New England Journal of Medicine, vol. 364, no. 9, pp. 883-885, 2011.

[18] M. G. Nikolaidis and A. Z. Jamurtas, "Blood as a reactive species generator and redox status regulator during exercise," Archives of Biochemistry and Biophysics, vol. 490, no. 2, pp. 7784, 2009.

[19] J. Zielonka, H. Zhao, Y. Xu, and B. Kalyanaraman, "Mechanistic similarities between oxidation of hydroethidine by Fremy's salt and superoxide: stopped-flow optical and EPR studies," Free Radical Biology and Medicine, vol. 39, no. 7, pp. 853-863, 2005.

[20] M. J. Jackson, R. H. T. Edwards, and M. C. R. Symons, "Electron spin resonance studies of intact mammalian skeletal muscle," Biochimica et Biophysica Acta, vol. 847, no. 2, pp. 185190, 1985.

[21] R. S. Richardson, A. J. Donato, A. Uberoi et al., "Exerciseinduced brachial artery vasodilation: role of free radicals," American Journal of Physiology, vol. 292, no. 3, pp. H1516H1522, 2007.

[22] C. K. Sen, "Oxidants and antioxidants in exercise," Journal of Applied Physiology, vol. 79, no. 3, pp. 675-686, 1995.
[23] R. R. Jenkins, "Free radical chemistry. Relationship to exercise," Sports Medicine, vol. 5, no. 3, pp. 156-170, 1988.

[24] C. E. Cooper, N. B. Vollaard, T. Choueiri, and M. T. Wilson, "Exercise, free radicals and oxidative stress," Biochemical Society Transactions, vol. 30, no. 2, pp. 280-285, 2002.

[25] L. M. A. Heunks, J. Viña, C. L. A. Van Herwaarden, H. T. M. Folgering, A. Gimeno, and P. N. R. Dekhuijzen, "Xanthine oxidase is involved in exercise-induced oxidative stress in chronic obstructive pulmonary disease," American Journal of Physiology, vol. 277, no. 6, pp. R1697-R1704, 1999.

[26] Y. Hellsten, "Xanthine dehydrogenase and purine metabolism in man. With special reference to exercise," Acta Physiologica Scandinavica, Supplement, vol. 151, no. 621, pp. 1-73, 1994.

[27] M. Paolini, L. Valgimigli, E. Marchesi, S. Trespidi, and G. F. Pedulli, "Taking EPR "Snapshots" of the oxidative stress status in human blood," Free Radical Research, vol. 37, no. 5, pp. 503508, 2003.

[28] D. M. Bailey, I. S. Young, J. McEneny et al., "Regulation of free radical outflow from an isolated muscle bed in exercising humans," American Journal of Physiology, vol. 287, no. 4, pp. H1689-H1699, 2004.

[29] Y. Michailidis, A. Z. Jamurtas, M. G. Nikolaidis et al., "Sampling time is crucial for measurement of aerobic exerciseinduced oxidative stress," Medicine and Science in Sports and Exercise, vol. 39, no. 7, pp. 1107-1113, 2007.

[30] W. S. Waring, A. Convery, V. Mishra, A. Shenkin, D. J. Webb, and S. R. J. Maxwell, "Uric acid reduces exercise-induced oxidative stress in healthy adults," Clinical Science, vol. 105, no. 4, pp. 425-430, 2003.

[31] T. A. Watson, R. Callister, R. D. Taylor, D. W. Sibbritt, L. K. Macdonald-Wicks, and M. L. Garg, "Antioxidant restriction and oxidative stress in short-duration exhaustive exercise," Medicine and Science in Sports and Exercise, vol. 37, no. 1, pp. 63-71, 2005.

[32] K. S. Echtay, T. C. Esteves, J. L. Pakay et al., "A signalling role for 4-hydroxy-2-nonenal in regulation of mitochondrial uncoupling," EMBO Journal, vol. 22, no. 16, pp. 4103-4110, 2003.

[33] H. M. Alessio, A. E. Hagerman, B. K. Fulkerson, J. Ambrose, R. E. Rice, and R. L. Wiley, "Generation of reactive oxygen species after exhaustive aerobic and isometric exercise," Medicine and Science in Sports and Exercise, vol. 32, no. 9, pp. 1576-1581, 2000.

[34] R. J. Bloomer, P. G. Davis, L. A. Consitt, and L. Wideman, "Plasma protein carbonyl response to increasing exercise duration in aerobically trained men and women," International Journal of Sports Medicine, vol. 28, no. 1, pp. 21-25, 2007.

[35] R. J. Bloomer, A. H. Goldfarb, and M. J. McKenzie, "Oxidative stress response to aerobic exercise: comparison of antioxidant supplements," Medicine and Science in Sports and Exercise, vol. 38, no. 6, pp. 1098-1105, 2006.

[36] C. Groussard, F. Rannou-Bekono, G. Machefer et al., "Changes in blood lipid peroxidation markers and antioxidants after a single sprint anaerobic exercise," European Journal of Applied Physiology, vol. 89, no. 1, pp. 14-20, 2003.

[37] R. R. Jenkins, "Exercise and oxidative stress methodology: a critique," American Journal of Clinical Nutrition, vol. 72, no. 2, supplement, pp. 670S-674S, 2000.

[38] N. B. J. Vollaard, J. P. Shearman, and C. E. Cooper, "Exerciseinduced oxidative stress: myths, realities and physiological relevance," Sports Medicine, vol. 35, no. 12, pp. 1045-1062, 2005.

[39] Y. J. Suzuki, M. Tsuchiya, and L. Packer, "Thioctic acid and dihydrolipoic acid are novel antioxidants which interact with 
reactive oxygen species," Free Radical Research Communications, vol. 15, no. 5, pp. 255-263, 1991.

[40] V. E. Kagan, A. Shvedova, E. Serbinova et al., "Dihydrolipoic acid-a universal antioxidant both in the membrane and in the aqueous phase. Reduction of peroxyl, ascorbyl and chromanoxyl radicals," Biochemical Pharmacology, vol. 44, no. 8, pp. 1637-1649, 1992.

[41] B. C. Scott, O. I. Aruoma, P. J. Evansi et al., "Lipoic and dihydrolipoic acids as antioxidants. A critical evaluation," Free Radical Research, vol. 20, no. 2, pp. 119-133, 1994.

[42] E. Busse, G. Zimmer, B. Schopohl, and B. Kornhuber, "Influence of $\alpha$-lipoic acid on intracellular glutathione in vitro and in vivo," Arzneimittel-Forschung, vol. 42, no. 6, pp. 829-831, 1992.

[43] R. Sumathi, V. K. Devi, and P. Varalakshmi, "DL $\alpha$-lipoic acid protection against cadmium-induced tissue lipid peroxidation," Medical Science Research, vol. 22, no. 1, pp. 23-25, 1994.

[44] T. A. Seaton, P. Jenner, and C. D. Marsden, "The isomers of thioctic acid alter 14C-Deoxyglucose incorporation in rat basal ganglia," Biochemical Pharmacology, vol. 51, no. 7, pp. 983-986, 1996.

[45] D. Yoshihara, N. Fujiwara, and K. Suzuki, "Antioxidants: benefits and risks for long-term health," Maturitas, vol. 67, no. 2, pp. 103-107, 2010. 


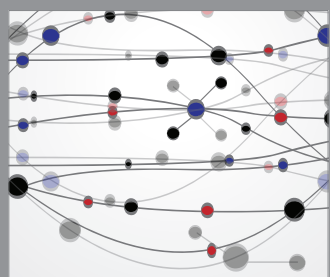

The Scientific World Journal
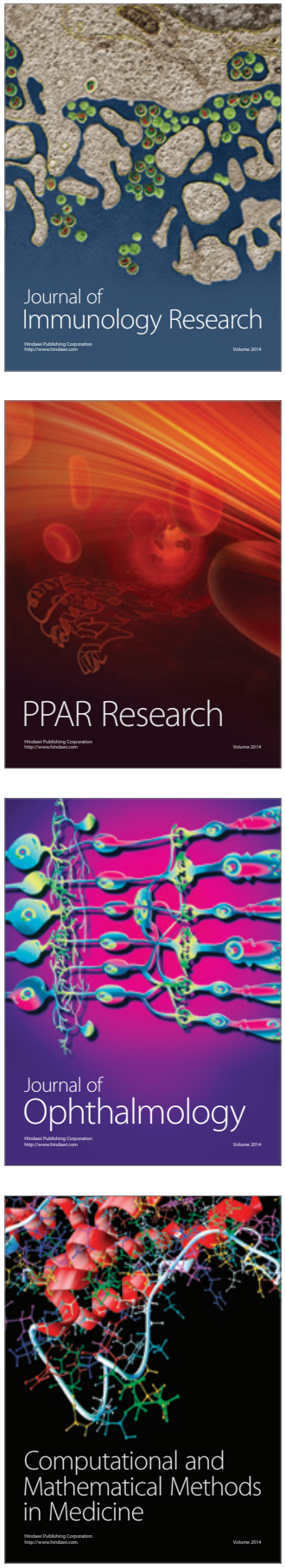

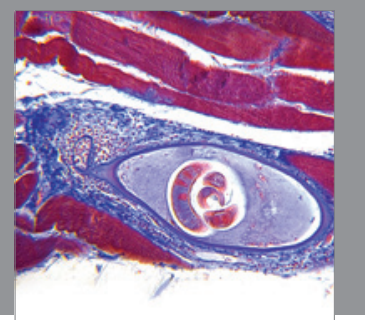

Gastroenterology

Research and Practice
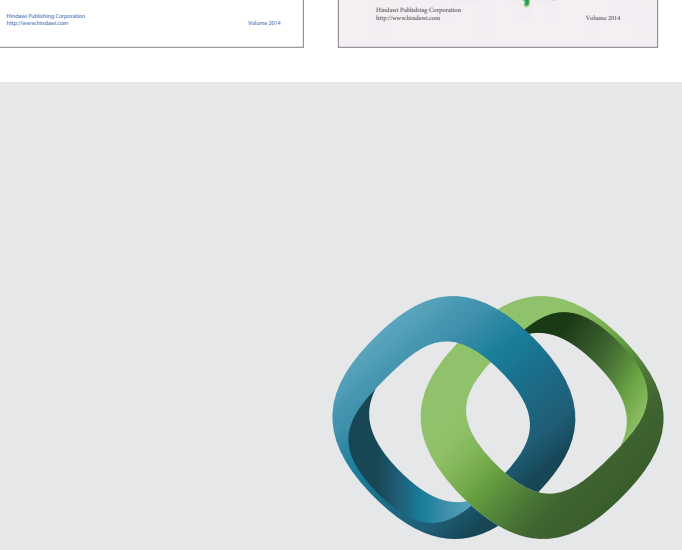

\section{Hindawi}

Submit your manuscripts at

http://www.hindawi.com
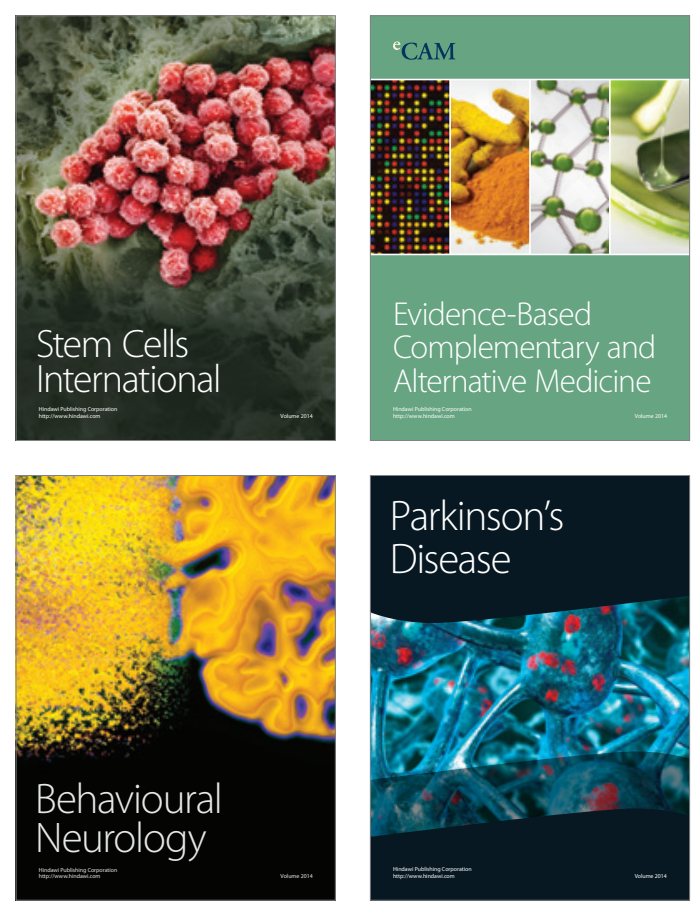

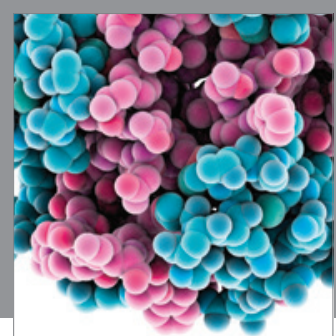

Journal of
Diabetes Research

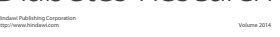

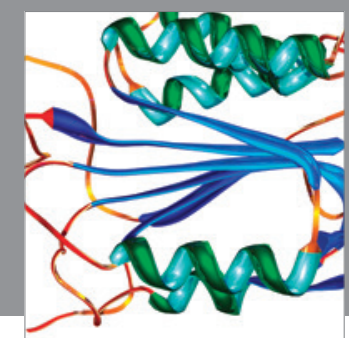

Disease Markers
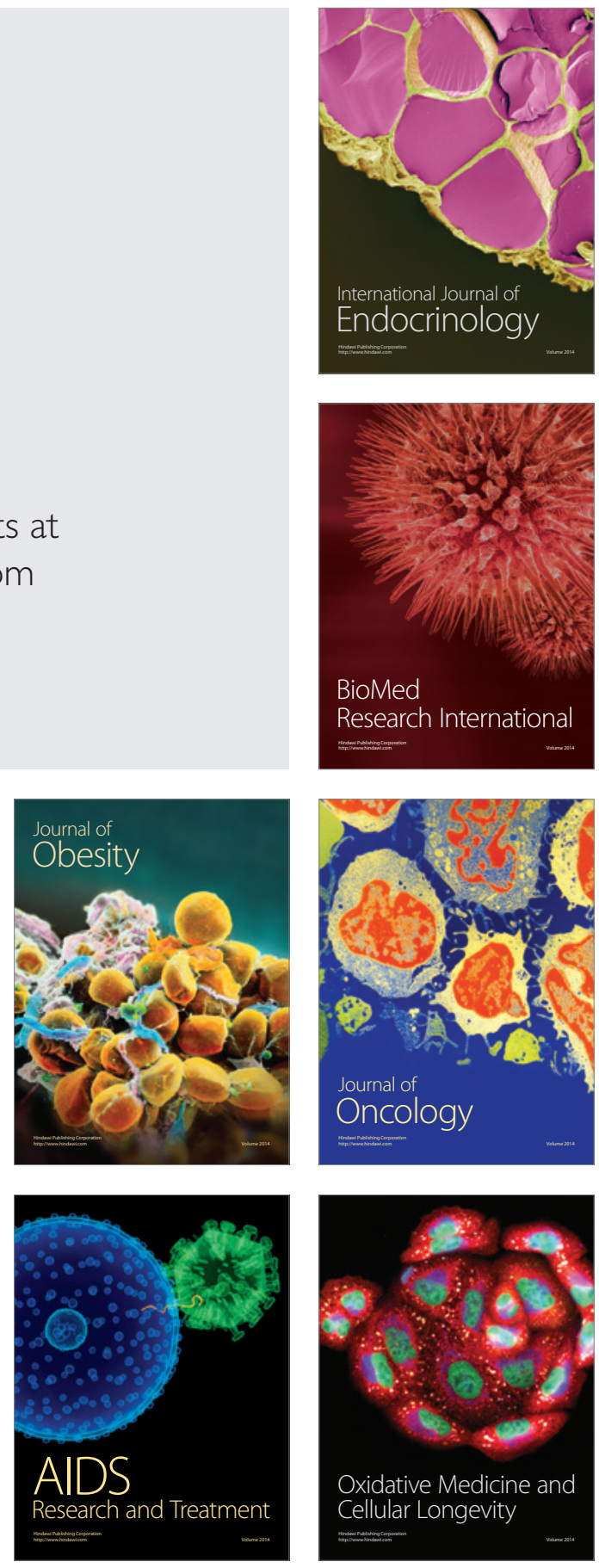